\title{
Minireview of drug safety and pharmacovigilance
}

\section{Nawaf Almuntashiri*}

Makkah Health Affairs, Ministry of Health, Saudi Arabia

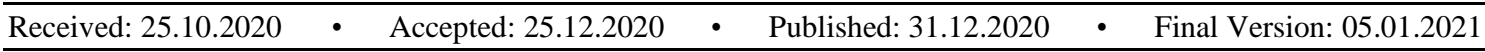

\begin{abstract}
Pharmacovigilance or drug safety is defined as science and actions related to diagnosis, evaluation, understanding and avoidance of harmful effects or any other problems related to drugs. Adverse drug reaction (ADR) may be caused by intrinsic factors, extrinsic factors, underlying medical conditions, interactions and wrong usage. Adverse drug reaction (ADR) may be caused by intrinsic factors, extrinsic factors, underlying medical conditions, interactions and wrong usage. ADR causing intrinsic factors include the active ingredients in the drug itself. In Saudi Arabia, the National Pharmacovigilance Center (NPC) was established in March, 2009 as a semi-autonomous department of Saudi Food and Drug Authority (SFDA). The purpose of NPC was to perform early detection of ADR and safe use of drugs. We need pharmacovigilance because animal experiments and clinical trials performed before marketing are insufficient evidence of drug safety.
\end{abstract}

Keywords: Pharmacovigilance, drug, clinical

\section{Introduction}

\subsection{Definitions and history}

Pharmacovigilance or drug safety is defined as science and actions related to diagnosis, evaluation, understanding and avoidance of harmful effects or any other problems related to drugs. It started on Jan 29, 1848 when a young girl died after administration of chloroform as an anesthetic prior to surgery of an infected toenail. She possibly died due to arrhythmia or pulmonary aspiration; however, exact reason of her death could not be identified. After several other cases of deaths due to anesthesia, The Lancet Journal made a commission to see this problem and doctor's reports about deaths caused by anesthesia were published in in this journal in 1983. In Jun 30, 1906, The Federal Food and Drug Act (FDA), US was established which focused on synthesis of only contamination free drugs and in 1911, FDA prohibited untrue drug indications. When sulfanilamide elixir caused 107 deaths in USA in 1937, the Federal Food, Drug and Cosmetic Act was created in 1938. According to this Act, the public health system was modernized and protocols for drug safety were established. All the companies were bound to allow any factory inspections and required to prove safety before launching a drug to the market. In 1961, congenital malformations of babies $(>20,000$ cases) due to thalidomide was reported and debated and this changed the system of Pharmacovigilance as reporting of adverse drug reactions became very systematic and regulated (Fornasier et al., 2018).

\footnotetext{
* Corresponding Author: nino_4789@ @otmail.com
} 


\subsection{Adverse drug reaction}

Adverse drug reactions (ADRs) are defined as any harmful, accidental and undesired effects of drugs that occur at doses recommended for diagnosis, prevention and treatment. ADRs were originally classified as Type A and Type B ADRs. Type A ADRs are predictable and dosedependent. They are also known as Augmented ADRs because they include augmentation of a known drug effect. Example includes orthostatic hypotension caused by antihypertensive drugs. Type B ADRs include unpredictable and dose-independent reactions that affect a small population e.g., hypersensitivity to drugs. Type B ADRs are also known as bizarre. Later on, 2 more types were added to ADRs including Type $\mathrm{C}$ and $\mathrm{D}$ that include chronic ADRs. Type $\mathrm{C}$ includes chronic reactions related to both time and dose while Type $\mathrm{D}$ includes delayed chronic reactions. Hypothalamic-pituitary-adrenal axis suppression by corticosteroids and teratogenesis are the examples of Type C and D, respectively. Recently two more categories including Type E and Type F have been included which refer to withdrawal and failure of therapy ADRs, respectively. Examples of Type E and F ADRs include withdrawal syndrome with opiates and resistance to antimicrobials, respectively (Schatz \& Weber, 2015).

\subsection{The possible cause of adverse drug reaction}

Adverse drug reaction (ADR) may be caused by intrinsic factors, extrinsic factors, underlying medical conditions, interactions and wrong usage. ADR causing intrinsic factors include the active ingredients in the drug itself. For example, non-steroidal anti-inflammatory drugs (NSAIDs) that are widely used as pain relievers may cause a wide range of adverse effects, the most common of which is peptic ulcer. However, the extrinsic factors include the characteristics of product that results from poor manufacturing, wrong formulation and poor-quality control i.e., contamination or adulteration. For example, a compounded drug, L-carnitine syrup, was reported to contain a dark fiber which was found to be Penicillium sp. after microbial analysis (Visacri et al., 2015). Assessment of ADR depends on number of patients exposed to drugs, relative frequencies of drug related and non-drug related events, methods used for assessment of toxicity and mechanism of toxicity. Clinical trials are generally conducted in few hundreds of patients on short term basis. Therefore, only acute ADRs can be detected that makes ADR detection inadequate in clinical trials (Naranjo, Busto \& Sellers, 1982).

\subsection{The establishment of Saudi Pharmacovigilance}

In Saudi Arabia, the National Pharmacovigilance Center (NPC) was established in March, 2009 as a semi-autonomous department of Saudi Food and Drug Authority (SFDA). The purpose of NPC was to perform early detection of ADR and safe use of drugs. The Yellow Card scheme is UK system for gathering and analyzing information on suspected drug safety concerns. It collects information about patient details, type of ADR, treatment provided, date, duration, history of patients, seriousness of ADR, if ADR disappears after stopping the drug, if ADR reappears on re-administration of drug, patient hospitalization due to ADR, para-clinical findings, patient suspected drugs and list of all the drugs taken at the time of ADR. NPC accepts reports from drug manufacturing companies, healthcare professionals, consumers and patients through the Saudi vigilance online reporting system, post message, e-mail, phone or fax. Several hospitals in the Kingdom have established Medication Safety Units that collect ADR data and report it to NPC. NPC shares this data with WHO-Uppsala Monitoring Center in Sweden (Ali et al., 2018). 


\subsection{Why do we need a Pharmacovigilance?}

Pharmacovigilance is aimed at detection, understanding, assessment and prevention of adverse drug effects. We need pharmacovigilance because animal experiments and clinical trials performed before marketing are insufficient evidence of drug safety. Dying from a disease may be expected but dying from a drug is undesirable. It allows evaluation of the adverse events that cross the line of drug's efficacy. In other words, it helps evaluate which side effects are worth to risk the patients as compared to effectiveness for treating a disease. If risk is more than the effectiveness, the drug may be removed from the market. Various drugs have been withdrawn form market as a result of pharmacovigilance strategies. For example, thalidomide was removed from global market in 1965 due to teratogenicity. Gemtuzumab ozogamicin which was used for treatment of leukemia but it was found to accelerate death. Therefore, it was withdrawn form USA market in 2010. In 2011, gatifloxacin was removed from the markets of Europe and India because it caused dysglycemia as ADR. Another example includes tetrazepam that was removed from the markets of Europe in 2013 because it causes dermatitis (Onakpoya, Heneghan \& Aronson, 2016).

\subsection{Pharmacovigilance cycle}

Pharmacovigilance cycle involves collection of data followed by analysis of data. Data related to safety of medicines is appropriately collected and managed. Any ADR signals in the data are analyzed and evaluated. Then a decision is made with regard to safety and suitable action is taken to protect public health. Actions include communication with stake holders i.e., to the manufacturing companies and warning may be issued. In rare cases it may include removal of drugs from the market to protect public safety (Lindquist, 2004). World Health organization has formed International Drug Monitoring Program that involves more than 123 countries across the globe. ADR reports from various countries are shared as Individual Case Safety Reports and submitted to the VigiBase system of this platform. All the data in VigiBase is detected and structured to reach solid conclusions. These conclusions are then used to make suitable decisions for the benefit of general public (Ali et al., 2018).

\subsection{Causality assessment and the Naranjo algorithm}

Causality assessment of ADR is a method to estimate the strength of relation between drug exposure and incidence of harmful reaction. One of the most widely used causality assessment test is Naranjo algorithm. Naranjo algorithm or adverse drug reaction probability scale is a method to evaluate if there is a causal relationship between an identified unpleasant clinical event and a drug using a questionnaire to assign probability scores. The questionnaire contains 10 questions that establish the likelihood if a reaction is caused by a drug. Each question in the questionnaire is weighted and total score is calculated to categorize a drug as unlikely, possible, probable and definite cause of ADR. It is often known as Naranjo scale and was developed by Naranjo and coworkers to standardize the evaluation of all ADRs in registration studies and controlled trials. However, it is simple to apply and therefore, widely used (Smyth et al., 2014).

\section{References}

[1]Ali, S., Egunsola, O., Al-Dossari, D. S., \& Al-Zaagi, I. A. (2018). Adverse drug reaction reporting in a large tertiary hospital in Saudi Arabia: results of an incentive strategy. Therapeutic advances in drug safety, 9(10), 585-590. 
[2]Fornasier, G., Francescon, S., Leone, R., \& Baldo, P. (2018). An historical overview over Pharmacovigilance. International journal of clinical pharmacy, 40(4), 744-747.

[3]Lindquist, M. (2004). Data quality management in pharmacovigilance. Drug Safety, 27(12), 857-870.

[4]Naranjo, C. A., Busto, U., \& Sellers, E. M. (1982). Difficulties in assessing adverse drug reactions in clinical trials. Progress in Neuro-Psychopharmacology and Biological Psychiatry, 6(4-6), 651-657.

[5]Onakpoya, I. J., Heneghan, C. J., \& Aronson, J. K. (2016). Post-marketing withdrawal of 462 medicinal products because of adverse drug reactions: a systematic review of the world literature. BMC medicine, 14(1), 10.

[6]Schatz, S., \& Weber, R. (2015). Adverse drug reactions. Pharmacy Practice, 1, 1. Available from https://www.accp.com/docs/bookstore/psap/2015B2.SampleChapter.pdf

[7]Smyth, R. L., Peak, M., Turner, M. A., Nunn, A. J., Williamson, P. R., Young, B., ... \& Conroy, E. J. (2014). ADRIC: Adverse Drug Reactions In Children-a programme of research using mixed methods. Available from https://www.ncbi.nlm.nih.gov/books/NBK262740/

[8] Visacri, M. B., de Souza, C. M., Sato, C. M. S., Granja, S., de Marialva, M., Mazzola, P. G., \& Moriel, P. (2015). Adverse Drug Reactions and quality deviations monitored by spontaneous reports. Saudi pharmaceutical journal, 23(2), 130-137. 